\title{
The Opossum genome reveals further evidence for regulatory evolution in mammalian diversification
} Bernardo Lemos

Address: Department of Organismic and Evolutionary Biology, Harvard University, Divinity Avenue, Cambridge, MA 02138, USA. Email: blemos@oeb.harvard.edu

Published: 9 August 2007

Genome Biology 2007, 8:223 (doi:10.1 I86/gb-2007-8-8-223)

The electronic version of this article is the complete one and can be found online at http://genomebiology.com/2007/8/8/223

(c) 2007 BioMed Central Ltd

\begin{abstract}
The sequencing of the euchromatic genome of a marsupial, the opossum Monodelphis domestica, identifies shared and unique features of marsupial and placental genomes and reveals a prominent role for the evolution of non-protein-coding elements.
\end{abstract}

Mammalian diversification began approximately 250 million years ago, yielding a stunning diversity of form and function that falls into three major groups with fundamentally distinct modes of reproduction. The monotremes (Prototheria) comprise a handful of extant species of egg-laying mammals: the platypus and a few species of echidna. Placentals (the Eutheria) comprise about 4,500-5,000 species characterized by lengthy gestation with placental nourishment and neonates born at an advanced developmental stage and often nearly independent from the mother. Marsupials (the Metatheria) comprise about 250-300 species that are characterized by a rudimentary placenta and short gestation, after which poorly developed embryos are born and undergo subsequent development during lactation, usually inside the mother's marsupium, or pouch.

Marsupial and placental mammals diverged about 125-190 million years ago [1-3]. While the marsupial radiation stopped short of producing as many species as the placental one, the impressive diversification of metatherians in South America and Australia resulted in a wide array of life histories and morphologies, many of which matched those observed in placental mammals - from herbivores to insectivores to carnivores. Two of the most notable cases are now extinct. The Tasmanian wolf (Thylacinus), also known as the pouched dog for its striking resemblance to placental canids, was heavily hunted and the last individual died in captivity in an Australian zoo in September 1936 [4]. Similarly, the marsupial Thylacosmilus from the South American Miocene possessed skull and saber-tooth morphologies with a remarkable resemblance to those of placental saber-tooth tigers [5]. Other cases of striking convergence include living species of marsupial 'anteaters', 'mice', 'moles', and 'flying squirrels' [5]. This remarkable natural experiment in mammalian evolution offers unique opportunities to understand the genomic and developmental correlates of these morphological convergences between marsupials and placentals. Most strikingly, these similarities in adult structures and lifestyles evolved on a background of fundamental differences in gestation and lactation, which imposed major shifts in the sequence and rate at which different body structures and organs develop in placentals and marsupials.

In a major step forward in understanding mammalian evolution, Mikkelsen et al. [6] recently completed the sequencing, assembly and annotation of the euchromatic segment of the first metatherian genome, that of the gray short-tailed opossum from South America, Monodelphis domestica (Figure 1). Their findings reveal a number of shared and unique features of the opossum and placental genomes, highlight a suite of opportunities unique to the opossum, and suggest new avenues for exploring the genomic and life-history diversity of marsupials.

\section{Monodelphis, a pouch-less marsupial}

The species chosen as the first marsupial genome to be sequenced does not resemble the popular marsupial picture of a large kangaroo with a big pouch. Indeed, $M$. domestica completely lacks a pouch, and the name of the genus, Monodelphis or 'single womb', alludes to this (delphi is Greek for womb). This is in contrast to the opossum genus 


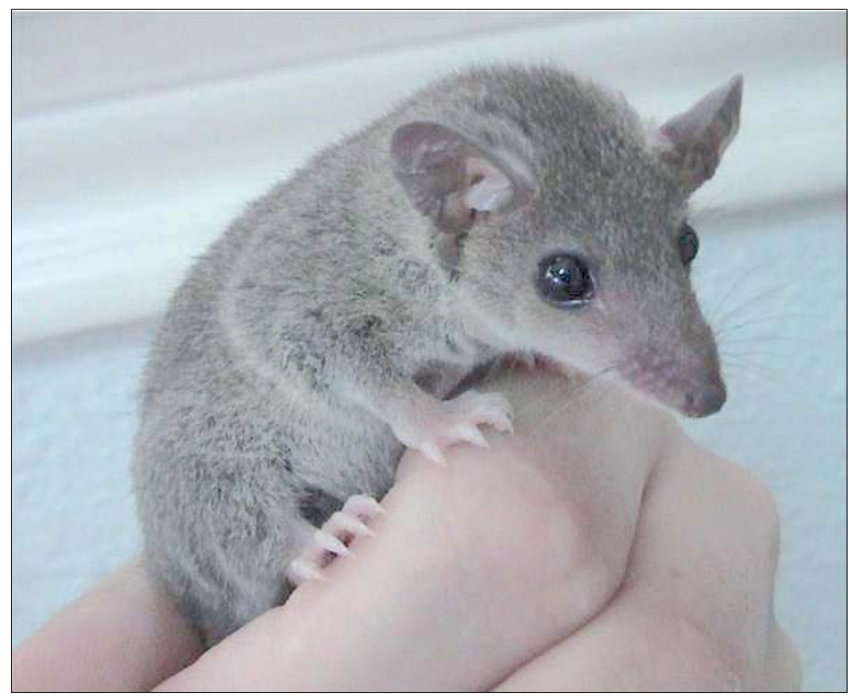

Figure I

The gray short-tailed opossum Monodelphis domestica.

Didelphis ('two wombs'), first described by Linnaeus in 1758 , which has an internal womb and external marsupium. $M$. domestica is, however, a convenient model marsupial: it can fit in the palm of the hand, breeds well in the laboratory and has been used as a model system in various areas of research [7]. A pregnant female gives birth to about 10 neonates after approximately 14-15 days of gestation, which is followed by a much longer lactation period of approximately 60 days. In newborns, hind limbs, eyes and ears, immune system and reproductive tissues are particularly poorly developed (altricial), the gonads are still undifferentiated and endothermy has not yet developed [8-11]. On the other hand, the bones and muscles of fingers, forelimbs and shoulders are well developed (precocious), as is the functional feeding apparatus of the mouth and stomach [8-11].

Such a mosaic of altricial and precocious traits matches the marsupial requirement to crawl from the birth canal to the pouch (or belly region), attach to the teats, and suckle without the help of the mother and after an extremely short period of intrauterine gestation (Figure 2). Indeed, birth at such an early stage of development followed by a lengthy lactation is a distinctive feature of marsupials compared with placental mammals, and has led to major differences in the timing and rate at which different body structures and organs develop in these two groups.

Hence, the publication of the opossum genome [6] offers opportunities both to inform the biology of placental mammals, including humans, and to foster a new era of opossum research. This may help enlighten us to the differences between marsupials and placentals and further our understanding of the biological underpinnings of unique and convergent traits evolved in these two groups. In the case of

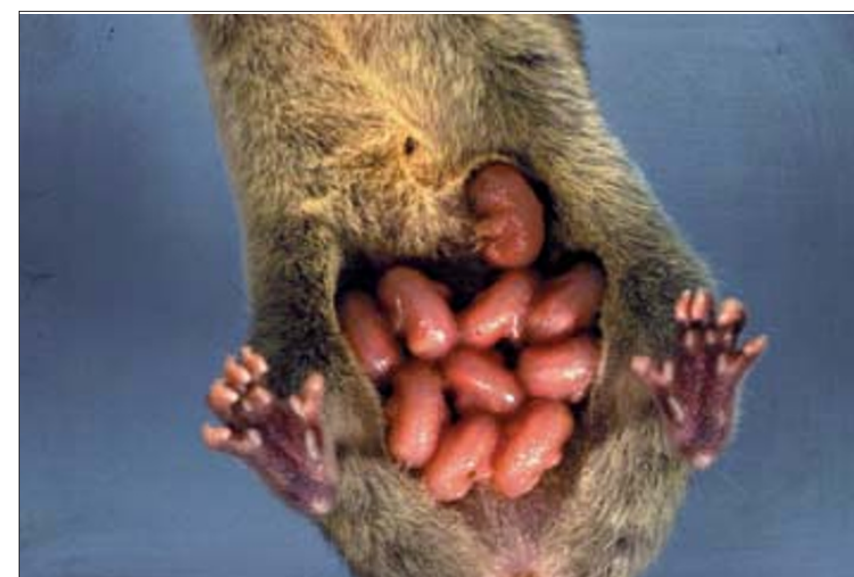

Figure 2

M. domestica with seven-day-old progeny attached to the mother's belly. Reproduced with permission [2I].

opossums and placentals this will certainly entail comparisons of organisms that are vastly different in terms of early embryogenesis, gestation, lactation and maternal care, although not so different in adult lifestyle and specific adaptations.

\section{Noncoding regions pinpoint differences}

There are many questions to be asked when comparing the opossum genome with that of placental mammals. How are protein-coding and regulatory sequences conserved between marsupials and placentals? What is the genomic composition of the opossum in terms of GC content and distribution of transposable elements? What are the roles of single-gene duplications and larger segmental duplications in shaping the structure of the two genomes? Are the effects homogeneous across chromosomes? How different are the mammalian and placental $\mathrm{X}$ chromosomes? How complex is the opossum immune system?

The publication of the opossum genome [6] and its companion papers provide initial answers to many of these questions. Perhaps the most striking result of the study of Mikkelsen et al. [6] is the apparently high turnover of regulatory elements between opossum and placental genomes, in sharp contrast to the conservation of proteincoding sequences. Accordingly, Mikkelsen et al. [6] find that only $1 \%$ of the eutherian coding sequences identified in human, mouse, and dog seem to be absent from the opossum. On the other hand, they estimate that around $20 \%$ of the noncoding elements conserved among eutherians are absent from the opossum. However, some of these elements might be particularly small and sensitive to detection methods, such that a higher false-negative rate might be expected in the distantly related opossum. To avoid this bias, Mikkelsen et al. [6] restricted their analysis to conserved noncoding elements that are long (average length 197 nucleotides) and 
unambiguously conserved within the Eutheria: among those elements they find that around $8 \%$ are not present in M. domestica [6]. Nevertheless, this still represents a substantial innovation in noncoding DNA in placentals, compared with the opossum, that is clearly beyond the innovation observed in protein-coding genes. Moreover, a substantial fraction of these eutherian-specific conserved noncoding elements reside close to genes with key developmental roles, suggesting that at least some of these elements are likely to underlie placental- and marsupialspecific developmental requirements. The implication of these findings is that divergence in noncoding DNA may be the sequence correlate of the various differences in the timing and rate of development of homologous body parts between placentals and marsupials.

These regulatory elements are inferred to have evolved within the lineage leading to the placentals after its split with the marsupial lineage. Although the uniqueness of these sequences among dogs, humans and mice, with no evidence of them in marsupials or the chicken, is suggestive of their evolution at the base of the placental lineage, it would not be too surprising if genome sequencing of other marsupials together with monotreme sequences revealed a more complex picture of lineage-specific gain and loss of particular elements. This will soon be put to the test, as the sequencing and assembly of additional genomes of placental mammals, marsupials and monotremes is under way [12].

On the other hand, the study by Mikkelsen et al. [6] points to an interesting example of how gene regulation might shape the evolution of chromosome structures and the sequences therein. In X inactivation in female marsupials it is always the paternal $\mathrm{X}$ chromosome that gets inactivated, whereas $\mathrm{X}$ inactivation is random in female placentals. Analyses confirm the expectation that the evolution of the $\mathrm{X}$-inactive specific transcript (XIST), which initiates X-chromosome silencing, occurred subsequent to the marsupial-placental split and presumably concomitantly with the evolution of random $\mathrm{X}$ inactivation in the placental ancestor $[6,13]$. Remarkably, the noncoding Xist RNA gene appears to have evolved from a protein-coding gene [13] and one hypothesis is that the evolution of XIST and random X inactivation led to a greater constraint on large-scale $\mathrm{X}$-chromosome organization in placental mammals compared to marsupials [6]. Mikkelsen et al. [6] suggest that a correlate of this is the higher density of LINE-1 transposons within conserved regions of the $\mathrm{X}$ chromosomes of placental mammals compared to the opossum, which presumably aids the spreading of $\mathrm{X}$-chromosome inactivation from the XIST center [14]. This could potentially explain the substantial conservation of $\mathrm{X}$-chromosome synteny in eutherians. It will be interesting to see how stable the $\mathrm{X}$ chromosome is within marsupials.

On a different note, Mikkelsen et al. [6] made the intriguing discovery that the opossum genome is substantially richer in
AT than previously sequenced amniote genomes (chicken, dog, mouse and human). This is interpreted to result from a reduced ability of GC-biased gene conversion to counterbalance AT-biased mutation rate in the opossum [15]. The plausibility of this interpretation arises from linkage data showing that recombination within opossum autosomes is substantially lower than that observed in other amniotes, as well as observations that the opossum has lower rates of gene and segmental duplication [6]. Hence, lowered recombination rates in opossum are interpreted to result in less gene conversion and, consequently, in a shift in nucleotide base composition towards AT. Further evidence for this hypothesis is provided by the $\mathrm{X}$ chromosome, in which the recombination rate is most similar to that observed in placentals and the GC content is almost twice that in opossum autosomes and well within the GC range observed in placental X chromosomes.

One temptation when interpreting marsupial-placental dichotomies is to take the marsupial state as ancestral or somehow more primitive. Difficulties in the cloning of opossum homologs of human immunity-related genes, such as those in the major histocompatibility complex (MHC), led to early suggestions that marsupials possessed a primitive immune system, with a level of complexity matching that of fish and birds. This notion has surely been dispelled by the publication of the opossum genome [6] and some of its companion papers $[16,17]$, which find that the complexity of the opossum immune system matches that of placental mammals. The difficulties in identifying and cloning marsupial MHC genes were possibly due to a high rate of innovation and divergence in genes of the immune system.

Finally, it is worth remembering that, as in many other eukaryotic genomes [18], large heterochromatic regions remain unsequenced in the opossum genome. These segments defy conventional methods of sequencing and assembly and understandably lag behind the determination of euchromatic segments. They include, for example, Y-chromosome sequences that remain an enormous challenge to sequence analyses in many species, including mammals [19] and flies [20]. A similar situation also occurs within other heterochromatic regions, such as those found within centromeres. Thus, it is fair to say that the physical gap represented by all heterochromatic regions of all genomes is matched by a knowledge gap of the genes and functional regions within them. Eventual elucidation of these segments will help further our understanding of the role of heterochromatin in eukaryotic genomic structure and regulation.

\section{Towards an integrative mammalian biology}

The opossum genome [6] highlights two central themes in eukaryotic genome evolution. First, it stresses the similarity of the complement of protein-coding genes across a large number of eukaryotic genomes. Second, it emphasizes the 
prominent role that differential expression of this protein set has on shaping physiological, morphological and behavioral differences across taxa. Hence, differences in the timing, location and expression level of a common set of genes appear to have led marsupials and placentals to take distinct paths towards developing a mammal, radically different in many aspects of gestation, embryogenesis and lactation.

What is most intriguing, however, is that many marsupials have evolved life histories and morphological adaptations that are strikingly similar to those evolved independently by placental mammals. Uncovering the regulatory changes underlying these major differences, together with those instances of striking convergence, promises to be fascinating. It can only be made more interesting and potentially informative with the addition of new genomes, some of which are already on their way, including those of a monotreme (the platypus Ornithorhynchus anatinus) and another marsupial (the tammar wallaby Macropus eugenii). In particular, $M$. domestica and the tammar wallaby are roughly the same evolutionary distance from each other as humans are from mice. This makes these two pairs ideally suited for providing valuable insights into the patterns of structural and regulatory genome evolution within and between each of these groups. Eventually, the integration of sequence data with phenotypic information will be crucial to interpreting the findings from comparative genome analyses. Relevant phenotypic information includes, for instance, genome-wide chromatin and gene-expression data with anatomical and developmental resolution that will inform both the biology and radiation of opossums as well as our own.

\section{Acknowledgments}

Thanks to David A Hewitt and Juliette J Selb for comments on the manuscript.

\section{References}

I. Ji Q, Luo ZX, Yuan CX, Wible JR, Zhang JP, Georgi JA: The earliest known eutherian mammal. Nature 2002, 416:816-822.

2. Kumar S, Hedges SB: A molecular timescale for vertebrate evolution. Nature 1998, 392:917-920.

3. Woodburne MO, Rich TH, Springer MS: The evolution of tribospheny and the antiquity of mammalian clades. Mol Phylogenet Evol 2003, 28:360-385.

4. Owen D: Tasmanian Tiger: The Tragic Tale of How the World Lost its Most Mysterious Predator. Baltimore: John Hopkins University Press; 2003.

5. Kirsch JAW: The classification of marsupials with special reference to karyotypes and serum proteins. In The Biology of Marsupials. Edited by Hunsaker D. New York: Academic Press; 1977: I-50.

6. Mikkelsen TS, Wakefield MJ, Aken B, Amemiya CT, Chang JL, Duke S, Garber M, Gentles AJ, Goodstadt L, Heger A, et al.: Genome of the marsupial Monodelphis domestica reveals innovation in non-coding sequences. Nature 2007, 447:167-177.

7. Samollow PB: Status and applications of genomic resources for the gray, short-tailed opossum, Monodelphis domestica, an American marsupial model for comparative biology. Aust | Zool 2006, 54: 173-196.

8. Tyndale-Biscoe H: Life of Marsupials. Collingwood: CSIRO Publishing; 2005.
9. Tyndale-Biscoe H, Renfree M: Reproductive Physiology of Marsupials. Cambridge: Cambridge University Press; 1987.

10. Vaglia JL, Smith KK: Early differentiation and migration of cranial neural crest in the opossum, Monodelphis domestica. Evol Dev 2003, 5:121-135.

II. Smith KK: Craniofacial development in marsupial mammals: developmental origins of evolutionary change. Dev Dyn 2006, 235: $1181-1193$.

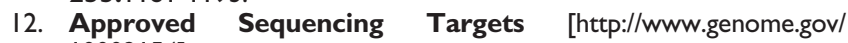
10002154]

13. Duret L, Chureau C, Samain S, Wessenbach J, Avner P: The Xist RNA gene evolved in eutherians by pseudogenization of a protein-coding gene. Science 2006, 3 I 2: 1653-I655.

14. Bailey JA, Carrel L, Chakravarti A, Eichler EE: Molecular evidence for a relationship between LINE-I elements and $X$ chromosome inactivation: The Lyon repeat hypothesis. Proc Natl Acad Sci USA 2000, 97:6634-6639.

15. Duret L, Eyre-Walker A, Galtier N: A new perspective on isochore evolution. Gene 2006, 385:7I-74.

16. Belov K, Deakin JE, Papenfuss AT, Baker ML, Melman SD, Siddle HV Gouin N, Goode DL, Sargeant T], Robinson MD, et al:: Reconstructing an ancestral mammalian immune supercomplex from a marsupial major histocompatibility complex. PLOS Biol 2006, 4:e46.

17. Belov K, Sanderson CE, Deakin JE, Wong ES, Assange D, McColl KA, Gout A, de Bono B, Barrow AD, Speed TP, et al.: Characterization of the opossum immune genome provides insights into the evolution of the mammalian immune system. Genome Res 2007, 17:982-991.

18. Carvalho AB, Vibranovski MD, Carlson JW, Celniker SE, Hoskins RA, Rubin GM, Sutton GG, Adams MD, Myers EW, Clark AG: $Y$ chromosome and other heterochromatic sequences of the Drosophila melanogaster genome: how far can we go? Genetica 2003, II 7:227-237.

19. Repping S, van Daalen SK, Brown LG, Korver CM, Lange J, Marszalek JD, Pyntikova T, van der Veen F, Skaletsky H, Page DC, Rozen S: High mutation rates have driven extensive structural polymorphism among human Y chromosomes. Nat Genet 2006, 38:463-467

20. Carvalho AB, Dobo BA, Vibranovski MD, Clark AG: Identification of five new genes on the $Y$ chromosome of Drosophila melanogaster. Proc Natl Acad Sci USA 200 I, 98: I 3225-I 3230.

21. Sakaguchi DS, Van Hoffelen SJ, Young MJ: Differentiation and morphological integration of neural stem cells transplanted into the developing mammalian eye. Ann NY Acad Sci 2003 995:127-139. 